\title{
ACTIVIDAD SÍSMICA EN COSTA RICA DURANTE EL 2012
}

\author{
SEISMIC ACTIVITY IN COSTA RICA DURING 2012
}

\author{
Lepolt Linkimer*, Rafael Barquero, Alberto Vargas, Wilfredo Rojas, \\ Magda Taylor \& María C. Araya
}

\author{
Red Sismológica Nacional (RSN: UCR-ICE), Apdo. 214-2060, \\ San Pedro, Costa Rica \\ *Autor para contacto: lepolt.linkimer@ucr.ac.cr
}

(Recibido: 14/09/2013 ; aceptado: 26/11/2013)

\begin{abstract}
During 2012, the National Seismological Network (RSN: UCR-ICE) recorded 3455 local earthquakes and reported 260 felt events. This high level of seismicity was mainly the result of the aftershocks and triggered seismicity of the Samara earthquake on 5 of September (Mw 7,6). There were also 12 significant earthquakes with magnitude $\geq 5,0$. The majority $(90 \%)$ of earthquakes were shallow $(<30 \mathrm{~km})$ and $56 \%$ had low magnitude $(\mathrm{Mw} 3,0-$ $3,9)$. The subduction of the Cocos Plate beneath the Caribbean Plate and the Panama microplate generated 53\% of felt earthquakes. Local faults originated $45 \%$ of felt seismicity, mainly in the Central Valley, the Calero Island, and the Coto Brus regions. The highest intensity observed during 2012 was VII (Modified Mercalli, MM) in the Nicoya Peninsula and in the Zarcero-Naranjo region due to the Samara earthquake. Moreover, eight other earthquakes produced intensities of V (MM).

Keywords: Seismicity in 2012, Costa Rican seismic sources, Samara earthquake.

RESUMEN: Durante el 2012 la Red Sismológica Nacional (RSN: UCR-ICE) registró 3455 sismos locales de los cuales 260 sismos fueron sentidos por la población. Esta alta sismicidad se relaciona con la ocurrencia del terremoto de Sámara del 5 de setiembre (Mw 7,6), sus réplicas y la sismicidad inducida en otras fuentes sísmicas. Se percibieron en total 12 sismos con magnitudes $\geq 5,0$. La mayoría (90\%) de los sismos percibidos fueron superficiales $(<30 \mathrm{~km})$ y el 56\% tuvo una magnitud baja (Mw 3,0-3,9). El proceso de subducción de la placa del Coco bajo la placa Caribe y la microplaca de Panamá originó el 53\% de los sismos sentidos. Un $45 \%$ fueron originados por fallas locales principalmente en el Valle Central, Isla Calero y Coto Brus. La intensidad máxima observada en el 2012 fue de VII (Mercalli Modificada, MM) para la zona de la península de Nicoya y Zarcero-Naranjo debido al terremoto de Sámara. Además, ocurrieron ocho sismos que ocasionaron intensidades de hasta V (MM).

Palabras clave: Sismicidad del 2012, fuentes sísmicas de Costa Rica, Terremoto de Sámara.
\end{abstract}




\section{INTRODUCCIÓN}

Durante el 2012 la Red Sismológica Nacional (RSN: UCR-ICE) registró 3455 sismos locales (i.e., sismos ubicados dentro del territorio de Costa Rica, Fig. 1, Cuadro 1). De estos 260 sismos fueron reportados como sentidos por la población (Fig. 2, Cuadro 1). El año 2012 se convierte en el tercer año con más sismos sentidos desde 1986, tiempo durante el cual la RSN tiene un registro completo de los sismos sentidos por la población. Solamente los años 1990 y 1991 tuvieron más sismos sentidos que el año 2012, con 655 y 380, respectivamente. La gran cantidad de sismos de 1990 y 1991 estuvo relacionada con la ocurrencia de los terremotos de Cóbano (Mw 7,0), Alajuela (Mw 6,0) y Limón (Mw 7,7), sus réplicas y la sismicidad inducida por estos sismos en otras fuentes. En el caso del año 2012, la alta sismicidad se relaciona con la ocurrencia del terremoto de Sámara del 5 de setiembre $(\mathrm{Mw} 7,6)$, sus réplicas y la sismicidad inducida por este terremoto en otras fuentes sísmicas. Con excepción de los años 1990, 1991 y 2012, desde 1986 la cantidad anual de sismos sentidos ha fluctuado entre 27 y 116 sismos.

La Red Sismológica Nacional (RSN) fue creada mediante un convenio entre la Sección de Sismología, Vulcanología y Exploración Geofísica de la Escuela Centroamericana de Geología de la Universidad de Costa Rica (UCR) y el Área de Amenazas y Auscultación Sísmica y Volcánica del Instituto Costarricense de Electricidad (ICE). El centro de registro conjunto de la RSN se localiza en las instalaciones en la Escuela Centroamericana de Geología de la UCR. Los sismos localizados por la RSN durante el 2012, fueron registrados por una red sísmica que estuvo constituida durante ese año por 60 estaciones ubicadas en diferentes partes del territorio costarricense. La mayoría de estaciones contaron con sismómetros de banda ancha de tres componentes, pero además existieron algunas estaciones con sismómetros de período corto analógico. Asimismo, se consideran en el proceso algunas estaciones de Panamá y Nicaragua. La detección y localización de los sismos localizados durante el
2012 se realizó a través de los sistemas Seiscomp, Earthworm y Seisan. El procesado de rutina, esto es, la lectura de arribos de ondas, localización y cálculo de magnitudes, se llevó a cabo mediante el programa HYP (Lienert \& Havskov, 1995) y la última versión del software sismológico Seisan (Ottemöller et al., 2011). La lectura de arribos se realizó aplicando un sistema de calidad por pesos de 0 a 4 ( 0 corresponde a la menor incertidumbre). Para el proceso de localización se empleó un modelo de velocidades de la onda Primaria (P) de siete capas y se estimaron dos tipos de magnitudes: coda (duración, $\mathrm{Md}$ ) y momento (Mw).

\section{CARACTERÍSTICAS DE LOS SISMOS SENTIDOS}

A continuación se describe la sismicidad sentida durante el 2012 tomando en cuenta su distribución temporal y por profundidad, magnitud, origen y región geográfica.

\section{Distribución temporal}

El mes con mayor cantidad de sismos sentidos fue setiembre con 83 sismos sentidos (Fig. 3A). Los meses con la menor cantidad de sismos sentidos fueron enero y abril, en los que se reportaron solamente cinco. A partir del terremoto del 5 de setiembre, la sismicidad aumentó considerablemente. Desde enero a agosto, el número de sismos sentidos varió entre 5 y 15 . En cambio, desde setiembre a diciembre, el número de sismos sentidos fue mucho mayor y varió entre 20 y 83 (Fig. 3A). El promedio mensual de sismos sentidos para el año 2012 fue de 22 .

\section{Distribución por profundidad}

El 90\% de los sismos sentidos tuvieron profundidades menores a $30 \mathrm{~km}$ (Fig. 3B). La mayoría de los sismos tuvieron profundidades menores a 9,9 km (106 sismos) y entre 10 y 19,9 km (93 sismos). Solamente 25 sismos sentidos tuvieron 


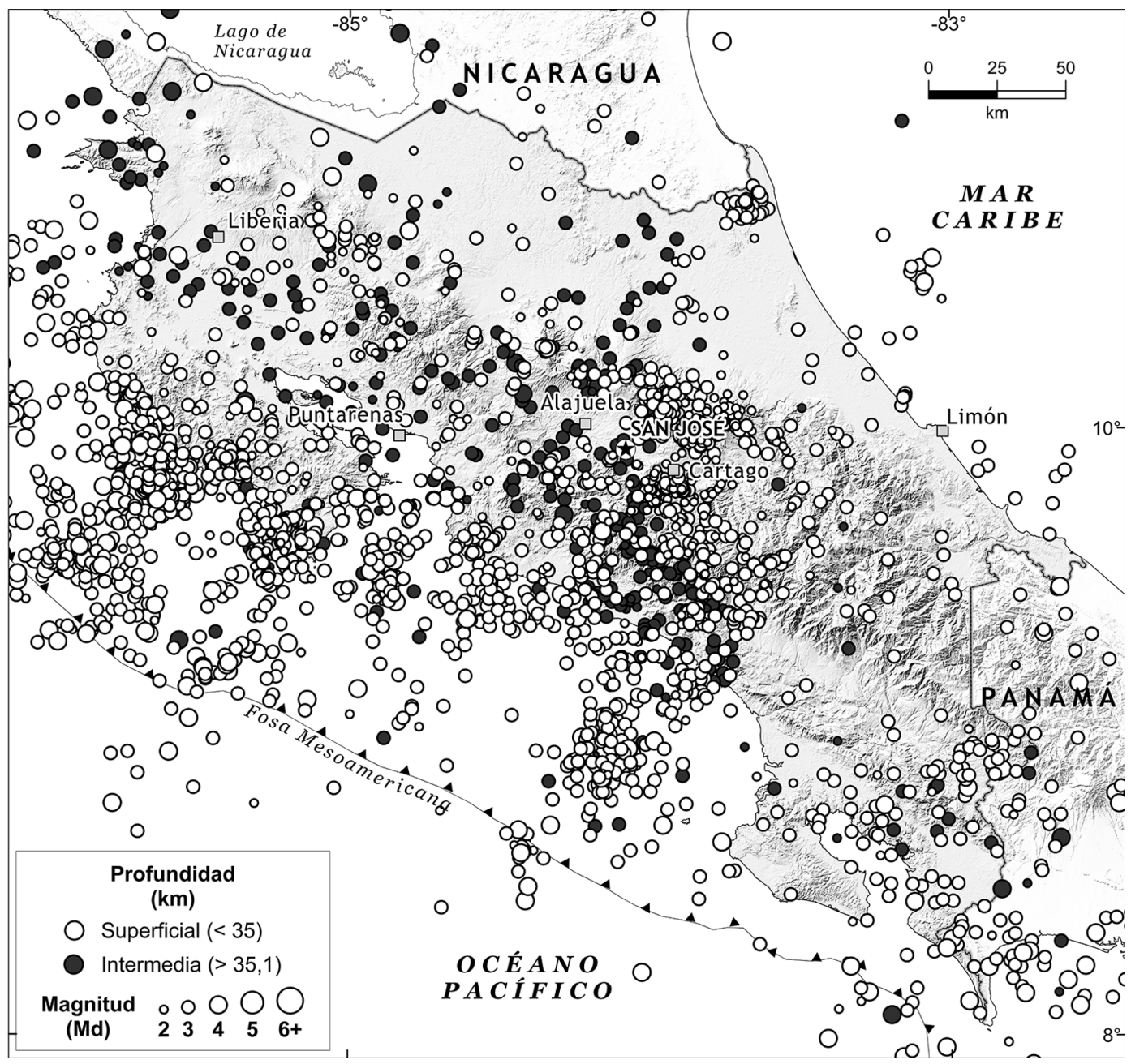

Fig. 1: Ubicación epicentral de los sismos localizados por la RSN durante el año 2012.

su hipocentro a más de $30 \mathrm{~km}$ de profundidad. Estos sismos están asociados a la deformación interna de la placa del Coco que se subduce debajo de Costa Rica. Es común que los sismos de profundidad mayor a $30 \mathrm{~km}$ no sean percibidos por la población a menos que sean de una magnitud intermedia o alta, ya que la energía se atenúa a lo largo del viaje de las ondas sísmicas desde un hipocentro profundo hasta la superficie. Destacan en el 2012 cuatro sismos de profundidad intermedia: el 17 de marzo a $90 \mathrm{~km}$ de profundidad localizado a $8 \mathrm{~km}$ al sur de La Tigra de San Carlos (Mw 3,7), el 7 de agosto a $93 \mathrm{~km}$ de profundidad ubicado a $5 \mathrm{~km}$ al oeste de Zarcero (Mw 4,5), el 16 de setiembre a $98 \mathrm{~km}$ de profundidad ubicado a $4 \mathrm{~km}$ al suroeste de la Virgen de Sarapiquí (Mw $3,3)$ y el 30 de marzo a $127 \mathrm{~km}$ de profundidad ubicado a $9 \mathrm{~km}$ al suroeste de La Fortuna de San Carlos (Mw 4,6).

\section{Distribución por magnitud}

El sismo de mayor magnitud del 2012 fue el terremoto de Sámara del 5 de setiembre, con una magnitud (Mw) de 7,6. El segundo sismo de 
Cuadro 1

Número de sismos locales registrados por la RSN y sentidos por mes durante el 2012

\begin{tabular}{ccc}
\hline Mes & Sismos Registrados & Sismos Sentidos \\
\hline Enero & 84 & 5 \\
Febrero & 184 & 13 \\
Marzo & 235 & 14 \\
Abril & 241 & 5 \\
Mayo & 248 & 7 \\
Junio & 218 & 15 \\
Julio & 105 & 10 \\
Agosto & 168 & 9 \\
Setiembre & 970 & 83 \\
Octubre & 529 & 40 \\
Noviembre & 262 & 39 \\
Diciembre & 211 & 20 \\
\hline TOTAL & 3455 & 260 \\
\hline
\end{tabular}

mayor tamaño, fue una réplica del terremoto del 5 de setiembre y esta ocurrió el día 23 de octubre y tuvo una magnitud (Mw) de 6,1. El tercer sismo más grande ocurrió el 6 de marzo, tuvo una magnitud (Mw) de 6,0 y se localizó $336 \mathrm{~km}$ al suroeste de Puerto Jiménez. El cuarto sismo más grande del 2012 fue el ocurrido el 13 de febrero de magnitud (Mw) 5,9 y localizado $40 \mathrm{~km}$ al sur de Quepos y el quinto sismo más grande ocurrió el 6 de marzo, tuvo una magnitud $(\mathrm{Mw})$ de 5,7 y se localizó a $130 \mathrm{~km}$ al suroeste de Puerto Jiménez. Los dos sismos ocurridos el 6 de marzo con magnitudes (Mw) de 6,0 y 5,7, fueron originados en la Zona de Fractura de Panamá y por localizarse tan alejados de la costa costarricense fueron percibidos de forma leve. En cambio, el sismo del 13 de febrero al sur de Quepos y el terremoto de Sámara del 5 de setiembre y su réplica más grande del 23 de octubre, fueron percibidos de forma fuerte y muy fuerte en las zonas epicentrales. En total, ocurrieron nueve sismos con magnitudes (Mw) entre 5,0 y 5,9.

El $32 \%$ de los sismos sentidos tuvieron magnitudes que oscilaron entre 4,0 y 4,9. La mayoría $(56 \%)$ de los sismos percibidos tuvieron una magnitud (Mw) de entre 3,0 y 3,9 (Fig. 3C). El sismo de menor magnitud del 2012 que fue reportado como sentido ocurrió el 1 de noviembre ( $\mathrm{Mw} 2,3)$ y se localizó a $2 \mathrm{~km}$ al sureste de Escazú. Este sismo fue percibido pese a su pequeña magnitud por ocurrir a poca profundidad y por estar localizado en una zona urbana. En total, 17 sismos pequeños (Mw $<2,9$ ) fueron percibidos por la población (Fig. 3C).

\section{Distribución por origen}

La mayoría de los sismos sentidos del 2012 (53\%) fueron originados por el proceso de subducción de la placa del Coco bajo la placa Caribe y la microplaca de Panamá (Fig. 3D). Este proceso incluye los sismos ocurridos en la zona interplacas, como el terremoto del 5 de setiembre y sus réplicas, así como los sismos de profundidad intermedia $(>50 \mathrm{~km})$, ocurridos debido a la deformación interna de la placa del Coco que se se subduce bajo Costa Rica, por ejemplo los cuatro sismos profundos mencionados anteriormente en el apartado de "Distribución por profundidad".

El $45 \%$ de los sismos del 2012 fueron originados por fallas locales en el interior de la placa Caribe y la microplaca de Panamá (Fig. 3D). Las zonas en donde se registró mayor actividad por fallamiento local fueron: Escazú, Desamparados y Cascajal de Vázquez de Coronado, en la provincia de San José; Zarcero de Alajuela; y, El Tejar de El Guarco, Orosi de Paraíso, Pacayas de Alvarado y Oreamuno en la provincia de Cartago. También hubo actividad asociada a fallamiento en Isla Calero de la provincia de Limón y en San Vito de Coto Brus de la provincia de Puntarenas. Adicionalmente, cuatro sismos fueron originados en la Zona de Fractura de Panamá, que corresponde con el límite entre las placas del Coco y Nazca.

\section{Distribución por región geográfica}

La mayoría de los sismos sentidos del 2012 ocurrieron en las provincias de Guanacaste (31\%), Puntarenas (28\%), Cartago (16\%) y San José $(12 \%)$ y en menor medida en las provincias de Alajuela (5\%), Limón (4\%) y Heredia (2\%). 


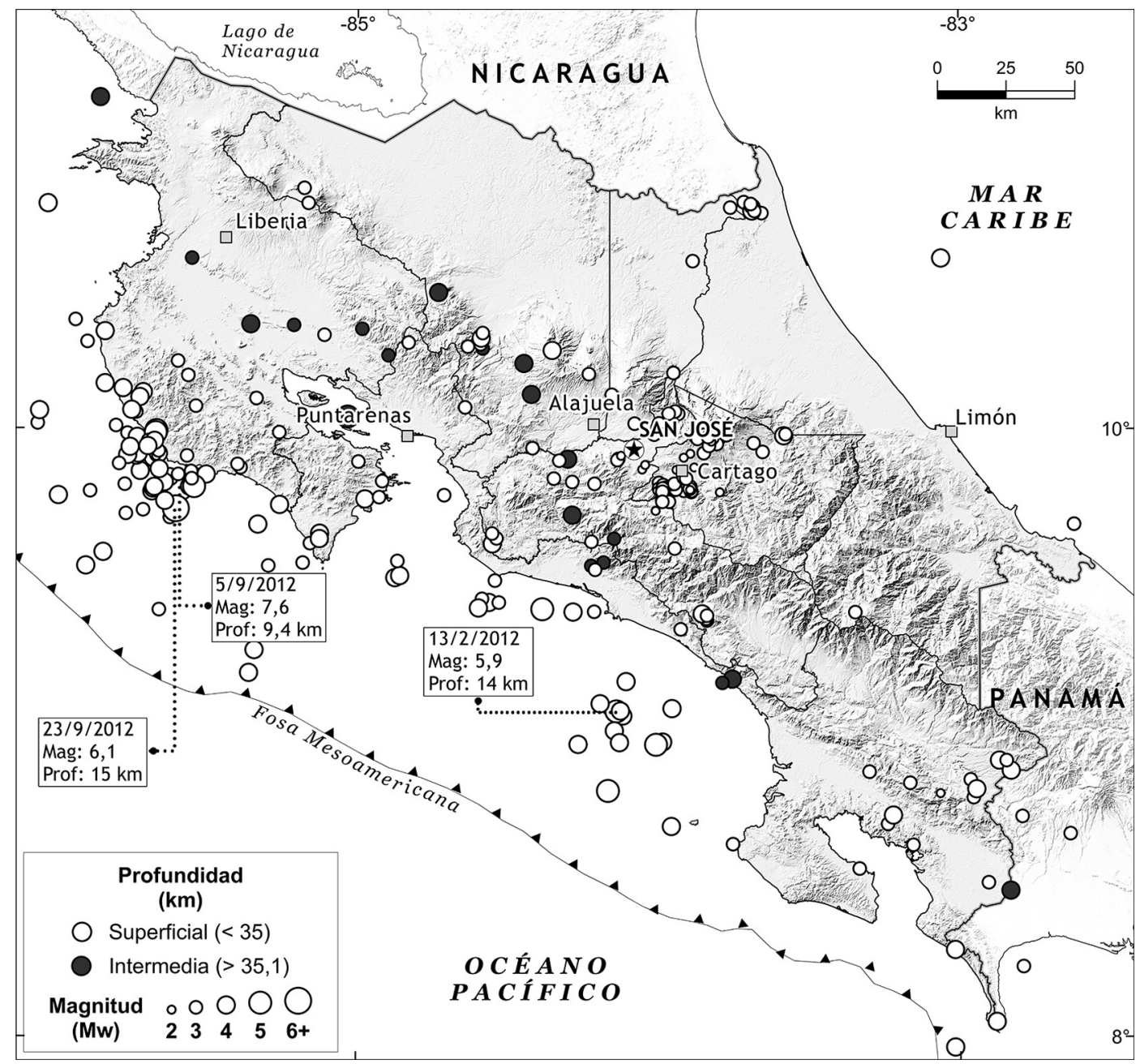

Fig. 2: Ubicación epicentral de los sismos sentidos durante el 2012 en Costa Rica.

Seis temblores (2\%) ocurrieron en los países vecinos de Panamá y Nicaragua pero fueron sentidos en el territorio costarricense. Se describen a continuación los sismos más importantes en cada una provincias de Costa Rica.

En Guanacaste se localizaron 82 sismos sentidos. En esta provincia ocurrió el terremoto de Sámara del día 5 de setiembre (Mw 7,6) y sus réplicas. Las cuatro réplicas de mayor tamaño ocurrieron los días 24 de octubre (Mw 6,1), 8 de setiembre (Mw 5,4), 15 de setiembre (Mw 5,2) y 10 de octubre (Mw 5,2). Antes del terremoto de Sámara los sismos más importantes que se presentaron en Guanacaste ocurrieron los días 2 de febrero al oeste de Tamarindo $(\mathrm{Mw} 4,4)$ y 19 de marzo en el golfo de Nicoya (Mw 5,0).

En la provincia de Puntarenas se registraron 73 eventos sísmicos sentidos algunos de los cuales tuvieron magnitudes importantes como el del día 13 de febrero de 2012 al sur de Quepos (Mw 5,9), el 12 de octubre en Parrita (Mw 5,1) y el día 13 de diciembre cerca de Dominical (Mw 5,4 y 5,5). En la zona Sur de la provincia de Puntarenas, se produjeron 30 sismos sentidos. La mayoría ocurrieron en los alrededores de Laurel de Corredores, Buenos Aires, Golfito y San Vito 


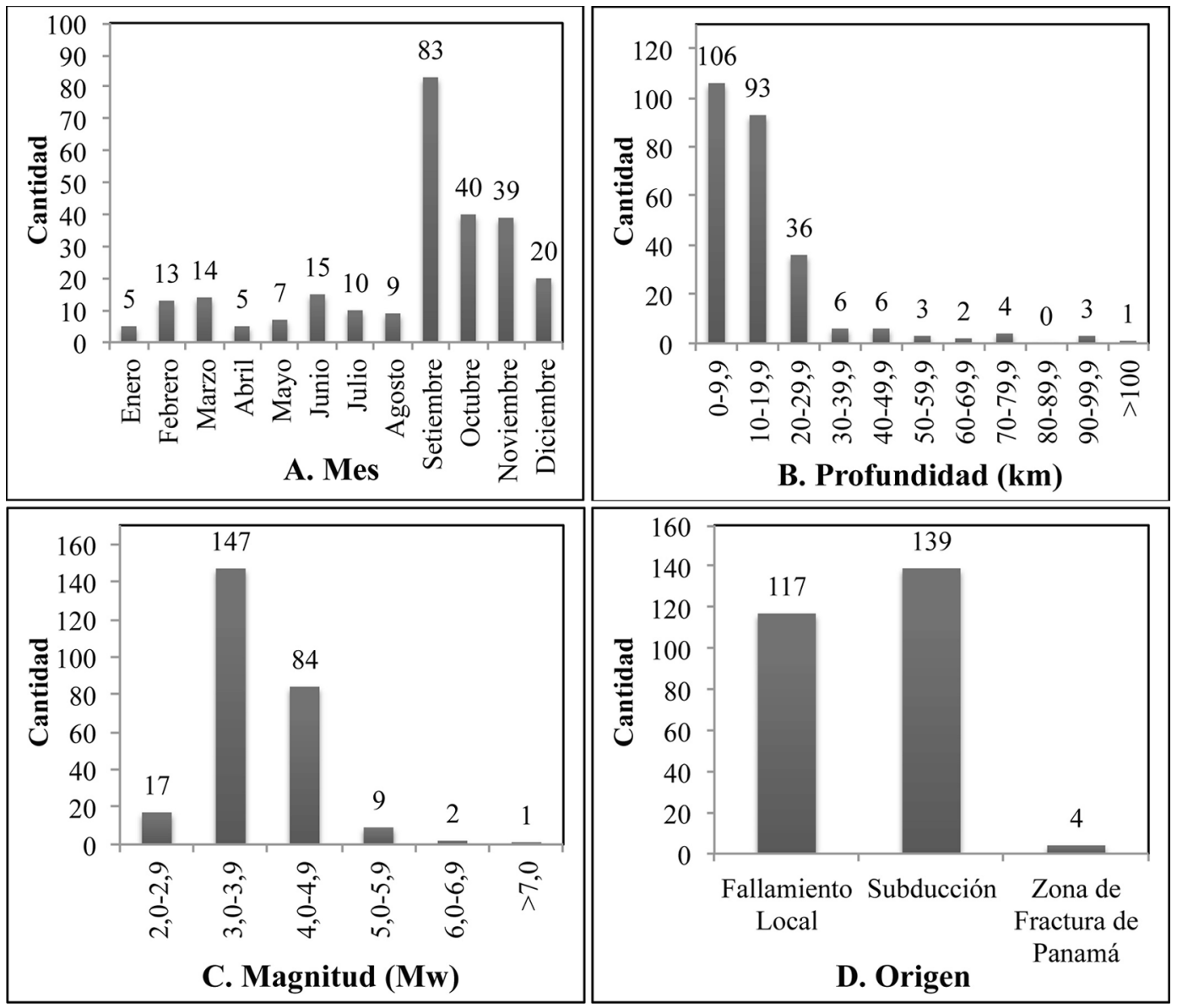

Fig. 3: Distribución de los sismos sentidos del 2012 por A) mes B) profundidad C) magnitud y D) origen. La cantidad de sismos se muestra sobre la barra de cada histograma.

de Coto Brus. Los sismos más grandes en esta región ocurrieron los días 26 de enero ( $\mathrm{Mw} 3,8)$, 12 de abril (Mw 4,3), 22 de julio (Mw 4,3), 28 de setiembre (Mw 4,2), el 15 de Octubre (Mw 4,1) y el 28 de diciembre (Mw 4,1).

En las provincias de Cartago y San José ocurrieron 41 y 30 sismos sentidos, respectivamente. De enero a setiembre, ocurrieron 13 sismos sentidos principalmente en las zonas de Desamparados, Tobosi y Aguacaliente de Cartago. Estos sismos tuvieron magnitudes bajas ( $\mathrm{Mw}<$ 3,6). Luego del terremoto de Sámara, tuvo lugar una reactivación de fallas en estas provincias reportándose 46 sismos sentidos ubicados en zonas como: Desamparados, Escazú, Puriscal, Cascajal de Vázquez de Coronado, Pacayas de Alvarado, Oreamuno, El Tejar de El Guarco, Orosi y Cachí de Paraíso y en las faldas de los volcanes Irazú y Turrialba (Mora et al., 2012).

En las provincias de Alajuela y Heredia se reportaron 13 y 5 sismos sentidos respectivamente. De estos se destacan los sismos profundos $(>$ $90 \mathrm{~km})$ que ocurrieron el 30 de marzo al suroeste de La Fortuna de San Carlos (Mw 4,6) y el 7 de 
agosto al oeste de Zarcero (Mw 4,5). Además, en la zona de Tapesco de Zarcero se ubicaron varios sismos superficiales $(<11 \mathrm{~km})$ ocurridos los días 19 y el 20 de setiembre (Mw 3,4-4,0).

En la provincia de Limón se reportaron 10 sismos sentidos. Luego del terremoto del 5 de setiembre ocurrió un enjambre de sismos con magnitudes (Mw) de entre 3,1 y 4,2 a lo largo del río Colorado, en la zona de Isla Calero, frontera con Nicaragua. Estos sismos son relevantes ya que esta zona es caracterizada por una sismicidad muy baja (Linkimer \& Rojas, 2012).

\section{Intensidades}

Las intensidades (Escala Mercalli Modificada, MM) más altas observadas durante el 2012 ocurrieron debido al terremoto del 5 de setiembre (Mw 7,6). La intensidad máxima fue de VII (MM) para la zona de la península de Nicoya y Puntarenas y además en Zarcero, Grecia y Naranjo (Linkimer et al., en este volumen) donde se presentaron efectos de amplificación debido a la geología local y la directividad de las ondas (Rojas, 2012). Además, durante el 2012 ocurrieron ocho sismos que ocasionaron intensidades de hasta V (MM). La réplica de mayor tamaño del terremoto de Sámara, ocurrida el 23 de octubre, produjo una intensidad máxima de $\mathrm{V}$ en la península de Nicoya, la ciudad de Puntarenas y además en Zarcero, Grecia y Naranjo. Los mapas de isosistas del terremoto de Sámara y su réplica principal son presentados por Linkimer et al. (en este volumen).

La intensidad $\mathrm{V}$ también fue alcanzada en localidades del Pacífico Central como Quepos y Parrita para los sismos ocurridos el 13 de febrero (Mw 5,9, Barquero, 2012), el 9 de junio (Mw 4,7) y el 12 de octubre (Mw 5,1). Además, se estima una intensidad de IV-V en sectores de la península de Nicoya para el sismo del 19 de marzo $(\mathrm{Mw} 5,0)$ y para las réplicas de mayor tamaño del terremoto de Sámara ocurridas el 8 de setiembre ( $\mathrm{Mw} 5,4), 15$ de setiembre (Mw 5,2) y 10 de octubre (Mw 5,2).

\section{AGRADECIMIENTOS}

Al personal de la RSN tanto de la Escuela Centroamericana de Geología de la Universidad de Costa Rica (UCR), como del Área de Amenaza y Auscultación Sísmica y Volcánica del Instituto Costarricense de Electricidad (ICE).

\section{REFERENCIAS}

BARQUERO, R., 2012: Informe de los sismos del 13 de febrero de 2012 (Mw 5,9) en el Pacífico Central de Costa Rica.- 15 págs. Instituto Costarricense de Electricidad [Inf. interno].

LIENERT, B.R. \& HAVSKOV, J., 1995: A Computer Program for Locating Earthquakes Both Locally and Globally, Seis. Res. Lett. 66: 26-36, DOI:10.1785/ gssrl.66.5.26.

LINKIMER, L. \& ROJAS, W., 2012: Sismicidad en la Isla Calero, Frontera de Costa Rica con Nicaragua.- En: LINKIMER. L. \& SOTO, G.J. (eds): El Terremoto de Sámara del 5 de setiembre del 2012.- Red Sismológica Nacional, San José [Inf. interno]: 81-83.

LINKIMER, L., ARROYO, I.G., MORA, M., VARGAS, A., SOTO, G. J., BARQUERO, R., ROJAS, W., TAYLOR, W. \& TAYLOR, M., 2013: El terremoto de Sámara (Costa Rica) del 5 de setiembre del 2012 (Mw 7,6).Rev. Geol. Amér. Central, 49: 73-82.

MORA, M.M., TAYLOR, W., \& SOTO, G.J., 2012: Sismicidad inducida y otros efectos causados en el arco magmático.- En: LINKIMER. L. \& SOTO, G.J., (eds): El Terremoto de Sámara del 5 de setiembre del 2012. Red Sismológica Nacional, San José [Inf. interno]:62-78. 
OTTEMÖLLER, L., VOSS, P. \& HAVSKOV, J., 2011: SEISAN: the Earthquake Analysis Software for Windows, Solaris, LINUX, and MACOSX, version 9.0.1.- 361 págs. Univ. of Bergen, Bergen.
ROJAS, W., 2012: Interpretación preliminar de la zona de ruptura y directividad de la energía.- En: LINKIMER. L. \& SOTO, G.J., (eds): El Terremoto de Sámara del 5 de setiembre del 2012. Red Sismológica Nacional [Inf. interno]: 41-42. 